\title{
Frontières
}

\section{Fascinant cadavre}

\section{Patrick Bergeron}

Volume 23, numéro 1, automne 2010

Enquêtes sur le cadavre : 1. Fascination

URI : https://id.erudit.org/iderudit/1004016ar

DOI : https://doi.org/10.7202/1004016ar

Aller au sommaire du numéro

Éditeur(s)

Université du Québec à Montréal

ISSN

1916-0976 (numérique)

Découvrir la revue

Citer ce document

Bergeron, P. (2010). Fascinant cadavre. Frontières, 23(1), 5-7.

https://doi.org/10.7202/1004016ar

Ce document est protégé par la loi sur le droit d'auteur. L'utilisation des services d'Érudit (y compris la reproduction) est assujettie à sa politique d'utilisation que vous pouvez consulter en ligne.

https://apropos.erudit.org/fr/usagers/politique-dutilisation/
Cet article est diffusé et préservé par Érudit.

Érudit est un consortium interuniversitaire sans but lucratif composé de l’Université de Montréal, l'Université Laval et l'Université du Québec à Montréal. Il a pour mission la promotion et la valorisation de la recherche. https://www.erudit.org/fr/ 
Patrick Bergeron

directeur du numéro

«La question Quelle sorte d'êtres sont les morts? est si insistante que même dans nos sociétés sécularisées nous ne savons pas quoi faire des morts, c'est-à-dire des cadavres. Nous ne les jetons pas aux ordures comme des déchets domestiques, que physiquement ils sont pourtant. L'imaginaire procède par glissement et généralisation: mon mort, nos morts, les morts » (Paul Ricœur, Vivant jusqu'à la mort suivi de Fragments, Paris, Seuil, coll. «La couleur des idées", 2007, p. 36-37).

Le cadavre est un objet à nul autre semblable. De lui émane une fascination entremêlée de répugnance. Le dégoût se comprend aisément: il procède de la révulsion avec laquelle nous accueillons tout ce qui évoque la décomposition, la putréfaction, le pourrissement. Les notions de «morbide», «macabre» ou «lugubre» n'ont guère la vertu de nous requinquer. Mais l'attirance s'explique tout aussi facilement: cet être que constitue le cadavre - nous répugnons résolument à en faire une chose -, cet être que la vie a déserté irrévocablement, ce résidu de la personne, nous savons (en général sans approfondir la question) que tôt ou tard, il sera nous - ou que nous serons lui (cette dissociation nous/lui étant habituellement maintenue) -, car tel est notre destinée post mortem. S'il y a un mystère de l'au-delà, dont sont nées les pensées eschatologiques, il n'y a pas de mystère de l'en deçà. Le cadavre est la conséquence prévisible, le résultat visible de la mort, la preuve concrète de la cessation d'être du point de vue physique. Nous sommes une espèce biodégradable.

Cette séduction ambivalente exercée par le cadavre est peutêtre plus active que jamais, aujourd'hui que nous avons érigé la vie en valeur suprême et que nous nous sommes dotés de normes et de lois pour préserver une distance convenable entre les défunts et nous. Ne visite pas une morgue qui veut. Le contact direct avec le cadavre, à moins qu'il ne soit légitimé par une pratique professionnelle, ne fait pas partie du quotidien du citoyen moyen. De plus, de tous les tabous à s'être formés au fil du temps dans notre civilisation, ceux qui touchent l'usage du cadavre, de l'anthropophagie à la nécrophilie, sont certainement parmi les plus tenaces en raison du malaise qu'ils remuent en nous.

Et pourtant, jamais le corps mort n'a occupé autant d'espace dans l'imaginaire.

Notre rapport au cadavre tend ainsi à se résumer à deux mots : surexposition et dissimulation. Nous sommes surexposés à des visions de cadavres. Jour après jour, la fiction populaire et les médias font défiler des visions de violence et d'horreur: homicides, guerres, tueries, catastrophes, suicides, accidents, génocides... Réels ou fictifs, ces événements funestes nous émeuvent sans doute, nous horripilent parfois, voire nous dévastent, mais ils ne nous surprennent plus. Une banalisation et une esthétisation du corps mort se sont mises en place qui se traduisent de multiples manières. Chaque jour apporte son bilan de victimes 
au journal télévisé, avec des images plus ou moins explicites. Tel jeu vidéo ou tel film d'action repousse les frontières du réalisme en montrant des corps mutilés, suppliciés, dépecés..

D'autres fois, notre surexposition au cadavre revêt des formes plus légères, plus inoffensives en quelque sorte, dénotant alors une omniprésence du cadavre à des endroits et sous des formes pour le moins insolites. Ce sera tantôt l'imagerie macabre dont s'inspire telle formation musicale rock, métal ou hip-hop; tantôt, ce sera tel accessoire courant de la mode, du sac à main au porteclés, que l'on verra orné de crânes ou d'ossements. C'est sans compter les collections fantastiques de la littérature jeunesse, la symbolique de l'Halloween en général ou l'actuel engouement des adolescents pour les histoires de vampires et de zombies... Sans toujours nous en rendre compte, nous sommes constamment approvisionnés en visions de cadavres.

Pourtant, il s'agit d'une familiarité trompeuse, car réels ou fictifs, anonymes ou personnalisés, tragiques ou comiques, ces cadavres sont avant tout imaginaires. À moins d'exercer une profession nous mettant en rapport avec des morts ou d'avoir été témoin d'un accident, d'un crime ou de tout autre événement du même ordre, nous n'avons que des images de cadavres. Nous vivons en effet à une ère de grande dissimulation post mortem. Les corps morts sont maintenus strictement hors de notre vue. L'usage de la veillée funèbre appartient à une époque révolue. De nos jours, on meurt à l'hôpital dans 80\% des cas. De nouveaux rituels et symboles sont venus médiatiser la fin de l'existence. Les endeuillés peuvent pleurer le cher disparu sans trop se soucier de sa dépouille. Le mort est pris en charge. Dans le cas de l'incinération, pratique désormais répandue, c'est le cadavre, littéralement, qui part en fumée.

Le cadavre est donc un objet sans pareil. Techniquement, il s'agit d'un déchet domestique. Dans les faits, il en va tout autrement. Les monuments aux morts font partie des manifestations les plus anciennes de civilisation humaine. C'est dire l'innéité de notre besoin de distinguer ce «déchet» du reste de la matière inerte.

Le seul terme de cadavre s'avère problématique. Tout indique qu'il fait davantage partie du vocabulaire des auteurs de polars que de celui des thanatologues ou des médecins-légistes. Ceux-ci préféreront parler de «corps », de «corps mort» ou de «dépouille».

Bref, le cadavre condense une bonne partie de notre résistance et de notre curiosité face à la mort. Il ne pouvait être que très instructif d'y consacrer un dossier entier.
Ce numéro spécial de Frontières a voulu enquêter sur la façon dont les sociétés humaines se positionnent devant la réalité du corps mort. En raison des multiples pistes que nos collaborateurs ont proposé d'explorer, nous avons vite constaté que l'entreprise prenait plus d'envergure que prévu. C'est pourquoi nous avons décidé de scinder notre enquête en deux volets. La première partie, qu'on lira ici, se charge d'étudier la fascination inspirée par le cadavre à l'homme. Elle sera suivie, dans le numéro subséquent de Frontières, d'une enquête sur la place du mort dans l'imaginaire fantastique.

Dans «Déclinaisons du cadavre, Esquisse anthropologique», David Le Breton soutient que le statut du cadavre est déterminé par des arguments culturels, une vision du monde ou une échelle de valeurs donnée. Une plongée dans l'histoire de l'anatomie lui permet d'opposer les brutalités du démembrement aux sensibilités culturelles, puis de faire ressortir ce qu'il appelle «l'humanité du cadavre ». L'impératif de contrôler sa vie, auquel chacun s'attache, s'applique dorénavant par-delà la mort, car l'on voit s'affirmer un souci de gérer la destination de son cadavre.

La question du corps et de son devenir post mortem se pose tout particulièrement dans le contexte de la pratique crématiste. Dans «Quels enjeux contemporains autour du cadavre et des cendres? », Gaëlle Clavendier examine la manière dont les lois et les normes professionnelles régissent les pra-

tiques funéraires contemporaines.

Dans «L'invasion néo-zombie: Entre l'abjection, le grotesque et le pathos (2002-2009) », Antonio Domínguez Leiva se penche sur le phénomène actuel de reviviscence du mort-vivant. Il montre que le zombie n'appartient plus au cinéma d'horreur ou gore des années 1960 et 1970, tel que l'ont emblématisé George A. Romero et Lucio Fulci, mais qu'il effectue un retour en force depuis les lendemains du 11 septembre. Cette «invasion néozombie» à laquelle nous assistons dans la littérature populaire a tellement pris d'ampleur que la critique universitaire s'est mise à s'y intéresser.

Dans «La contemplation du cadavre dans la méditation bouddhiste et les arts visuels », Karine Delage élucide deux pratiques au miroir l'une de l'autre: d'une part, la contemplation de charniers pour méditer sur la mort; d'autre part, l'intégration du cadavre dans certaines productions contemporaines en arts 
visuels. Toutes deux permettent de mieux comprendre la puissance d'évocation et le pouvoir symbolique du corps mort.

Dans «Le photographique mémoriel: Dire la mort et son contraire », Caroline Ziolko étudie l'identification et la commémoration des morts dans les cimetières, des débuts de la photographie aux nouvelles technologies. Elle montre que le photographique mémoriel a évolué parallèlement avec le développement de l'image du corps dans les médias de large diffusion.

Dans «Georges Bataille et la question du corps mort», Gilles Ernst nous fait comprendre que la hantise de la mort qui s'affirme chez Bataille est le support d'une description délibérément provocatrice. Bataille a moins été fasciné par la mort et les morts qu'il n'a développé à leur propos une conscience lucide. Il a pris le sujet très au sérieux, renouant en plein $\mathrm{XX}^{\mathrm{e}}$ siècle avec l'esthétique baroque. Il était grand temps de procéder à une lecture anthropologique de son œuvre.

Dans «La logique cannibale de Montaigne», Irène Salas et Flore Marie s'appuient elles aussi sur une anthropologie de la littérature. À partir du célèbre chapitre des Essais, «Des cannibales » (livre I, ch. XXXIII), elles démontrent que les Tupinambas ont permis à Montaigne de servir une contre-leçon de civilisation aux Européens. Au lieu d'y appréhender des mœurs inhumaines et barbares, l'humaniste aperçoit chez ces peuplades du Nouveau Monde un modèle d'organisation sociale très harmonieuse. L'article fait aussi ressortir l'importance du cadavre à la Renaissance, notamment comme source de connaissance et de sagesse dans la pensée humaniste.

Une autre époque a beaucoup fait progresser les connaissances sur le cadavre: le XIX ${ }^{\mathrm{e}}$ siècle, auquel se consacrent trois articles. Dans "L'autopsie médico-légale, spectacle cadavérique?", Sandra Menenteau retrace l'apparition d'une volonté de dissimulation dans l'expertise judiciaire française du XIX ${ }^{\mathrm{e}}$ siècle. Dans "L'autopsie à l'école de médecine navale de Rochefort», Grégory Bériet explique comment la mise en place d'un registre des autopsies, dans les années 1810, correspondait à une volonté d'approfondir les connaissances médicales sur le corps. Dans "Andro-lithe et pétrification des cadavres humains au XIX ${ }^{\mathrm{e}}$ siècle», Gian Marco Vidor s'intéresse à la transformation des tissus en pierre et à l'art des pétrificateurs français et italiens. Il s'agit d'une facette méconnue de l'histoire de la conservation des cadavres, puisque les médecins et les anatomistes ont eu tendance à l'assimiler à une «nouvelle alchimie».

Il aurait été difficile de conclure ce premier dossier d'enquête sans faire référence à la plastination, la controversée technique de préservation des tissus biologiques inventée par l'anatomiste allemand Gunther von Hagens. Jacques Brunet-Georget propose une réflexion sur le «cas» von Hagens au regard de la psychanalyse et du concept lacanien de «l'entre-deux-morts».

Une autre composante décisive de notre rapport contemporain au cadavre est le trauma provoqué par la shoah. À partir des mémoires de Claude Lanzmann, Le lièvre de Patagonie, et du roman Zone de Mathias Énard, Hans-Jürgen Greif propose une réflexion sur les répercussions intimes et les responsabilités individuelles dans le déchaînement du mal et de la barbarie. Le "cadavre apparent» semble mort, mais il continue à poser des questions chez le (sur)vivant que hantent des visions aux limites du supportable. Pour finir, Corinne Benestroff analyse la double modulation de l'univers concentrationnaire chez Jorge Semprún. Les camps nazis sont certes la démonstration du «Mal radical », mais ils sont aussi la possibilité inattendue d'un sauvetage de la fraternité.

Voilà peut-être en quoi réside le pouvoir de fascination du cadavre: nous rappeler que l'humanité reste avant tout une fraternité. 